\title{
Outcomes following transsphenoidal surgical management of incidental pituitary adenomas: a series of 52 patients over a 17-year period
}

\author{
Justin Seltzer, BA, ${ }^{1,3}$ Michelle A. Wedemeyer, MD, PhD, ${ }^{1,3}$ Phillip A. Bonney, MD,1 \\ John D. Carmichael, MD, 2,3 Martin Weiss, MD, ${ }^{1,3}$ and Gabriel Zada, MD ${ }^{1,3}$ \\ 1Department of Neurosurgery, Division of Endocrinology, ${ }^{2}$ Department of Medicine, and ${ }^{3}$ USC Pituitary Center, Keck School of
Medicine of USC, Los Angeles, California
}

\begin{abstract}
OBJECTIVE Incidental pituitary adenomas (IPAs) are commonly discovered during cranial imaging evaluations obtained for unrelated indications. The optimal management of IPA remains controversial. The authors investigated the outcomes and safety of the surgical treatment of IPAs at their institution.
\end{abstract}

METHODS Clinical outcome data for 1692 patients surgically treated for pituitary adenomas at the Keck Medical Center of USC/USC Pituitary Center over a 17-year period (1999-2016) were reviewed to identify all cases with surgically managed IPAs. Clinical characteristics reviewed in this retrospective analysis included patient demographics, endocrine laboratory data, visual field examinations, and MRI results. Intraoperative data reviewed included requirement for CSF leak repair, surgical complications, and estimated extent of resection. Postoperative data collected included pathology results, length of stay, postoperative complications, endocrine outcomes, readmission rates, and long-term outcomes, including extent of resection noted on postoperative imaging studies and tumor progression and/or recurrence.

RESULTS Fifty-two patients ( $3.1 \%$ of all cases) underwent transsphenoidal surgery for IPA. The median age at surgery was 61 years (range 31-86 years). The most common reasons for neuroimaging included trauma (19\%), stroke/transient ischemic attack (15\%), and sinonasal disease (15\%). Visual field deficits were present in $15 \%$ of bedside examinations, and among the 22 patients sent for formal testing, $54.5 \%$ were noted to have deficits. Preoperative endocrine function was normal in $69 \%$ of patients, which includes 3 patients $(5.8 \%$ ) having isolated hyperprolactinemia consistent with a stalk effect without other hormonal dysfunction. The average maximal tumor diameter was $20.9 \mathrm{~mm}(8-50 \mathrm{~mm}$; data available in 35 patients). The most common primary indication for surgery was compression of the chiasm or vision loss $(52 \%)$; other major considerations included tumor growth, a young patient age, and identified endocrine abnormalities. Intraoperative CSF leak repair was performed in $56 \%$ of patients, and 1 patient $(2 \%)$ developed postoperative CSF rhinorrhea treated with lumbar drainage. The median hospital stay was 2 days. There were no deaths or major complications. Three patients $(5.8 \%)$ developed transient diabetes insipidus. Over a mean follow-up of 61 months, 4 patients (50.0\%) reported improved headaches and $6(54.5 \%)$ reported improvement in their visual deficits. Four patients $(25 \%)$ had improved endocrine function, including one with resumption of menstruation and another with remission of acromegaly. One patient (2.4\%) reported new postoperative headache, and none experienced worsened vision. Four patients (10.5\%) developed new single-axis hypopituitarism and $1(2.6 \%)$ developed new panhypopituitarism. The overall recurrence/progression rate on neuroimaging was $9.6 \%$ at a mean of 80 months.

CONCLUSIONS Transsphenoidal resection of IPAs, when appropriate, can be performed safely at experienced treatment centers. Incidental pituitary adenomas should be evaluated and treated as indicated, especially in younger patients at risk for endocrine or visual dysfunction.

https://thejns.org/doi/abs/10.3171/2017.11.JNS171485

KEYWORDS transsphenoidal; incidental; adenoma; macroadenoma; pituitary surgery 
I NCIDENTAL pituitary adenomas (IPAs), or pituitary "incidentalomas," may be discovered during the course of evaluation for unrelated events or symptoms, including cancer staging, head trauma, or nonspecific complaints (e.g., dizziness).,19 These masses are relatively common; prior studies have reported an estimated prevalence of $3.7 \%-37 \%$ on imaging and $1.5 \%-27 \%$ on autopsy assessment. ${ }^{7,14,16}$ In general, the overall prevalence of incidentalomas is increasing as greater numbers of people undergo head CT or MRI as a part of routine evaluation for nonspecific complaints and head trauma. ${ }^{19}$

Pituitary adenomas (PAs) are among the most common masses found incidentally in the sella. In a surgical series of 911 incidental sellar masses, approximately $90 \%$ were PAs. ${ }^{6}$ A study of 1000 autopsy specimens revealed 178 incidental lesions, the most common of which were 37 Rathke's cleft cysts and 20 PAs. ${ }^{20}$ The most common subtype of IPA found at autopsy is the nonfunctional PA. ${ }^{16}$

A vast majority of IPAs are microadenomas $(<10 \mathrm{~mm})$ and remain clinically insignificant given the absence of both mass effect and hormonal dysfunction. ${ }^{16,19}$ Occasionally, however, IPAs become symptomatic, show subclinical evidence of hormonal hypo- or hypersecretion, result in subclinical vision loss, or demonstrate growth on repeat imaging studies and thus warrant operative intervention.

In this study, we assess indications for surgery, patient characteristics, complications, and clinical outcomes in patients treated surgically for IPAs at a high-volume tertiary care pituitary center over a $17-$ year period. We review our findings in the context of the published literature and discuss our treatment algorithm for these common lesions.

\section{Methods}

A single-institution database search was conducted to identify incidentally found PAs. Confirmation of diagnosis from surgical specimens was required for inclusion. Local approval from the University of Southern California (USC) institutional review board was granted prior to our review of patient data.

\section{Database Source and Construction}

The records of 1692 patients were obtained exclusively from the Keck Medical Center electronic medical record system using both internal records and scanned outside records. These data were then transcribed into the USC Pituitary Outcomes Database, a secure online database. Study data were collected and managed using Research Electronic Data Capture (REDCap) tools hosted at USC. ${ }^{9}$ REDCap is a secure, web-based application designed to support data capture for research studies, providing 1) an intuitive interface for validated data entry, 2) audit trails for tracking data manipulation and export procedures, 3) automated export procedures for seamless data downloads to common statistical packages, and 4) procedures for importing data from external sources. No paper charts were available at the time of transcription. Patient records were initially screened for various clinical criteria and labeled accordingly when entered into the database.

\section{Case Identification and Database Search}

During the data transcription process, cases were labeled as either incidental or nonincidental. An IPA was defined as a tumor diagnosed during the course of a workup for an unrelated or nonspecific complaint. Patients whose tumors were found in the course of evaluating complaints that had sellar pathology as a prominent differential diagnosis, such as headache, endocrinopathies, syndrome of inappropriate antidiuretic hormone (SIADH), diabetes insipidus (DI), or bitemporal hemianopia, were excluded. Once this process was completed, a search of the database was run to identify patients treated surgically for IPA. Clinical characteristics reviewed in the retrospective analysis included patient demographics, endocrine laboratory data, visual field examinations, and MRI results. Intraoperative data reviewed included requirement for CSF leak repair, surgical complications, and estimated extent of resection. Postoperative data collected included pathology results, length of stay, postoperative complications, endocrine outcomes, readmission rates, and long-term outcomes, including extent of resection noted on postoperative imaging studies and tumor progression and/or recurrence. Patients with significantly incomplete records were excluded, specifically those with unavailable postoperative radiology reports or follow-up documentation as these cases could not contribute to the outcome data.

\section{Patient Care Algorithm}

Patients were treated as part of a multidisciplinary center with pituitary specialists in neurosurgery, endocrinology, radiology, and radiation oncology. Baseline testing included MRI of the sella with and without gadolinium and endocrine testing of growth hormone $(\mathrm{GH})$, insulinlike growth factor 1 (IGF-1), morning cortisol or 24-hour urinary free cortisol, adrenocorticotrophic hormone (ACTH), thyroxine (T4), thyroid-stimulating hormone (TSH), follicle-stimulating hormone (FSH), luteinizing hormone (LH), testosterone, and prolactin. Hormonal deficiencies were corrected prior to surgery in consultation with endocrinology and with particular attention to thyroid or adrenal deficiencies. Patients with clinical or biochemical evidence of GH-secreting tumors underwent glucose tolerance testing. Patients with prolactin-secreting tumors had a trial of medical management with dopamine agonists, most commonly cabergoline. Surgery was firstline therapy for other secretory tumors.

Patients with nonfunctional microadenomas were typically offered conservative management with serial MRI. Patients with tumors abutting or displacing the optic chiasm and clear visual compromise on bedside testing were typically offered surgery, as were patients whose incidentally discovered prolactin-secreting tumors failed to respond to medical management. Those whose tumors abutted or displaced the optic chiasm but had normal vision on bedside testing were referred for formal visual field testing. Patients with evidence of visual compromise on formal ophthalmological examination were typically offered surgery. Young, healthy patients with macroadenomas not causing vision loss were given the option of surveillance with annual MRI and visual exams or surgery. Tumors 
with growth on serial imaging studies were typically managed operatively.

Patients were seen by our pituitary endocrinology group prior to surgery and were co-managed by neurosurgery and endocrinology in the perioperative period. First-line surgical treatment was transsphenoidal craniotomy. Patients were treated via a microscopic approach prior to 2011; thereafter, most patients were treated via an endoscopic approach. As the conclusion of tumor resection, intraoperative Valsalva maneuver was performed to detect CSF leaks. Such leaks were repaired with a fat and/ or fascial graft from the abdomen or thigh and reinforced with Duragen or Surgicel tucked under the edges of the sphenoid opening. Postoperatively, patients were observed overnight in the intensive care unit with strict attention to the neurological examination and fluid status. If no evidence of a CSF leak, hypopituitarism, or DI was observed, patients were discharged home on postoperative day 1 . Serum sodium was checked on an outpatient basis on postoperative day 5-7.

Magnetic resonance imaging with contrast of the sella was obtained at 3 months postoperatively, annually for 5 years, and then at 7 and 10 years postoperatively. Although our center is a referral center and many patients have their long-term endocrine care managed outside of our network, detailed medication reconciliations were performed at all outpatient visits to facilitate long-term monitoring of endocrine status.

Patients with significant residual tumor in the cavernous sinus or adjacent to the optic apparatus were referred to Radiation Oncology for CyberKnife treatment or, alternatively, an open craniotomy to address the suprasellar components.

\section{Results \\ Patient Demographics}

The database search identified 52 cases of pathologically verified IPAs treated via transsphenoidal operations between 1999 and 2016, representing 3.1\% of the total cases surgically treated over the same time period. This series included 27 male (52\%) and 25 female (48\%) patients. The median age at surgery was 61 years (range $31-86$ years). The median follow-up was 67 months, the mean was 61 months, and the range was 3-204 months.

\section{Indications for Neuroimaging in IPA}

The indications for neuroimaging resulting in an IPA diagnosis are listed in Table 1. The most common indications included trauma (19\%), workup for stroke/transient ischemic attack (15\%), and nasopharyngeal/sinus symptoms (15\%). Additional indications included cancer surveillance/evaluation $(10 \%)$, dizziness $(6 \%)$, seizure $(6 \%)$, hearing loss (4\%), neck pain (4\%), and whole-body scan (4\%), as well as altered mental status, memory loss, Bell's palsy, syncope, tremor, persistent periorbital edema, multiple sclerosis surveillance, anosmia, and meningioma follow-up $(<2 \%$ each $)$.

\section{Clinical Symptoms and Endocrine Findings}

Despite the incidental nature of the diagnosis, a de-
TABLE 1. Reasons for initial neuroimaging

\begin{tabular}{lc}
\hline \multicolumn{1}{c}{ Reason } & No. \\
\hline Trauma & 10 \\
\hline Cerebrovascular accident evaluation & 8 \\
\hline Sinonasal symptoms & 8 \\
\hline Neoplasia (surveillance/screening/evaluation) & 5 \\
\hline Dizziness & 3 \\
\hline Seizure evaluation (acute or chronic) & 3 \\
\hline Neck pain & 2 \\
\hline Whole-body scan & 2 \\
\hline Hearing loss & 2 \\
\hline Altered mental status & 1 \\
\hline Memory loss & 1 \\
\hline Bell's palsy & 1 \\
\hline Syncope & 1 \\
\hline Tremor & 1 \\
\hline Persistent periorbital edema & 1 \\
\hline Multiple sclerosis surveillance & 1 \\
\hline Anosmia & 1 \\
\hline Postop FU (meningioma removal) & 1 \\
\hline
\end{tabular}

$\mathrm{FU}=$ follow-up.

tailed history and physical examination revealed at least one nonspecific clinical symptom in $63.5 \%$ of patients (33/52). The most common symptoms included vision loss $(23 \%)$, headache $(21 \%)$, decreased libido (17\%), and fatigue (8\%; Table 2). One patient had signs and symptoms consistent with undiagnosed acromegaly. Detailed physical examination and endocrine testing were normal in $25 / 52$ patients $(48 \%)$.

A total of $16 / 52$ patients had visual loss on bedside examination or formal examination of visual fields. Formal ophthalmological visual field testing identified field cuts in $12 / 22$ patients (54.5\%) for whom exams were available for review. Of the 12 patients with a field cut on formal examination, patient history or bedside exam revealed findings in only $6(50 \%)$. Three patients reported visual loss that was ultimately attributed to the following nonpituitary pathologies: inadequate glasses prescription, hemangioma, and ocular migraine. One patient was found to have coincident bitemporal hemianopia from impingement of the chiasm and macular degeneration.

No evidence of hypopituitarism was seen in 36/52 patients (69.2\%), although 3 patients without evidence of hypopituitarism exhibited an isolated prolactin level $<150$ $\mathrm{ng} / \mathrm{ml}$ consistent with a pituitary stalk effect. Hormonal deficits included hypogonadism (7/52 [13\%]), primary hypothyroidism (5/52 [9.6\%]), pituitary hypothyroidism (5/52 [9.6\%]), hypocortisolemia (3/52 [6\%]), and GH deficiency (3/52 [6\%]). A total of 7/52 patients (13\%) exhibited elevated prolactin consistent with a stalk effect; $3 / 7$ had an isolated stalk effect with no other evidence of hypopituitarism, while the remaining 4/7 had other coincident hormonal deficits. One 40-year-old patient with a $10 \times 8-\mathrm{mm}$ tumor and a prolactin level of $113 \mathrm{ng} / \mathrm{ml}$ was initially presumed to have a stalk effect and was referred for surgery 
TABLE 2. Patient demographics and presentation

\begin{tabular}{|c|c|}
\hline Parameter & Value \\
\hline No. of patients & 52 \\
\hline Male & 27 \\
\hline Female & 25 \\
\hline \multicolumn{2}{|l|}{ Age in yrs } \\
\hline Median & 61 \\
\hline Range & $31-86$ \\
\hline Normal physical exam \& biochemical studies & 25 \\
\hline \multicolumn{2}{|l|}{ Associated symptoms } \\
\hline None & 19 \\
\hline Vision loss & 12 \\
\hline Headache & 11 \\
\hline Decreased libido & 9 \\
\hline Fatigue & 4 \\
\hline Dizziness & 3 \\
\hline Amenorrhea/oligomenorrhea & 1 \\
\hline \multicolumn{2}{|l|}{ Physical exam } \\
\hline Normal & 35 \\
\hline Any visual complaint & 16 \\
\hline Visual acuity loss (subjective) & $2 / 52$ \\
\hline Visual field cut (bedside exam) & $8 / 52$ \\
\hline Visual field cut (formal exam) & $12 / 22$ \\
\hline Acromegaly & 1 \\
\hline \multicolumn{2}{|l|}{ Preop endocrine status } \\
\hline Normal & $36^{*}$ \\
\hline \multicolumn{2}{|l|}{ Hyperprolactinemia } \\
\hline Stalk effect (<150 ng/ml) & $7 \dagger$ \\
\hline Prolactinoma & 2 \\
\hline Hypogonadism & 7 \\
\hline Primary hypothyroidism & 5 \\
\hline Secondary hypothyroidism & 5 \\
\hline Hypocortisolemia & 3 \\
\hline GH deficiency & 3 \\
\hline Acromegaly & 1 \\
\hline
\end{tabular}

* Includes 3 patients with prolactin level < $150 \mathrm{ng} / \mathrm{ml}$ consistent with an isolated stalk effect. The patients were counted as having a normal preoperative endocrine status.

$\dagger$ Three of 7 patients had an isolated prolactin level $<150 \mathrm{ng} / \mathrm{ml}$ consistent with a stalk effect and no other evidence of hormonal deficiency. Two of 3 patients had coincident visual deficit, whereas 1 of 3 had otherwise normal physical examination and biochemical findings.

because of her age; however, pathology was consistent with a prolactin-secreting tumor.

Three tumors were found to be functional/hypersecretory preoperatively. One patient was found to have an incidental GH-secreting macroadenoma. Physical examination was consistent with early symptoms of acromegaly, and biochemical testing revealed a poor response to glucose tolerance testing and an IGF- $1>700 \mathrm{ng} / \mathrm{ml}$. She was treated with resection and followed biochemically. Two patients presented with prolactinomas. As described above, one patient was initially classified as having a nonfunctioning pituitary adenoma because of a prolactin level of $113 \mathrm{ng} / \mathrm{ml}$; however, surgical pathology revealed a prolactinoma. She was asymptomatic at the last follow-up without the need for dopaminergic agonist therapy. The third patient was a 47-year-old shuttle driver who was found to have an incidentally discovered cystic Knosp stage IV pituitary mass with invasion into the hypothalamus and frontal lobe on a head CT obtained for trauma. History and physical examination revealed normal vision; however, he reported memory loss and long-standing hypogonadism managed with androgen supplementation. Further workup was significant for a prolactin level $>1000 \mathrm{ng} / \mathrm{ml}$ that recovered to $5.6 \mathrm{ng} / \mathrm{ml}$ after 10 months of treatment with cabergoline. Despite improvement in his hormone status, there remained substantial mass effect from the cyst in addition to personality changes, impaired memory, and seizures. The patient ultimately underwent transsphenoidal craniotomy, followed by radiation and an eyebrow craniotomy to address the suprasellar component. At $>4$ years from his initial surgery, the patient was neurologically intact without the need for hormonal supplementation beyond testosterone.

\section{Imaging Characteristics}

Forty-eight patients (92\%) had incidental macroadenomas $(>10 \mathrm{~mm}$ in diameter). One patient with a microadenoma $(<10 \mathrm{~mm}$ in diameter) and known breast cancer underwent surgery due to progressive growth and the need to rule out metastasis prior to cranial radiation therapy; pathology in this patient was consistent with a null cell adenoma. Three patients did not have a documented tumor size. The mean maximal tumor diameter was $20.9 \mathrm{~mm}$ (range $8-50 \mathrm{~mm}, 35$ patients with available measurement data). A majority of cases had suprasellar extension (81\%) and cavernous sinus invasion (56\%). Only three patients (6\%) had no MRI evidence of extrasellar extension or invasion.

\section{Indications for Surgery}

The most common indication for surgery was visual loss or compression of the optic chiasm (41/52 [79\%]), followed by growth on serial imaging studies (13/52 [25\%]), young age $(5 / 52[10 \%])$, hypopituitarism $(3 / 52[6 \%])$, and hypersecretion (2/52 [4\%]; Table 3$)$. One patient with a $2-\mathrm{cm}$ macroadenoma with invasion into the cavernous sinus but no suprasellar extension opted for resection over surveillance with annual MRI.

\section{Operative Management and Surgical Complications}

All 52 patients were surgically managed via a transsphenoidal approach. Operative data are detailed in Table 4. Fifty patients underwent microscopic transsphenoidal surgery, and 2 underwent endoscopic transsphenoidal surgery. Intraoperative CSF leak repair was performed in $56 \%$ of cases, with all but one receiving autologous fat grafting and $14(48 \%)$ receiving additional sellar floor reconstruction using a multilayer fascial apposition repair. One patient $(2 \%)$ required additional structural reinforce- 
TABLE 3. Indications for surgery

\begin{tabular}{lcc}
\hline \multirow{2}{*}{ Indication } & \multicolumn{2}{c}{ No. of Patients } \\
\cline { 2 - 3 } & Total & Sole Reason for Op \\
\hline Chiasm compression or vision loss & 41 & 27 \\
\hline Tumor growth & 13 & 4 \\
\hline Patient age $<60$ yrs & 5 & 3 \\
\hline Hypopituitarism & 3 & 1 \\
\hline Prolactinoma & 2 & 0 \\
\hline Acromegaly & 1 & 1 \\
\hline Patient preference & 1 & 1 \\
\hline
\end{tabular}

ment with collagen dural regeneration matrix. The median hospital stay was 2 days (range 2-7 days).

There were no serious intraoperative or postoperative adverse events, such as meningitis, carotid artery injury, significant hemorrhage, pulmonary embolism, pneumonia, paresis, significant vision loss, stroke, coma, or death. The most common complication was transient DI, which occurred in 3/52 patients $(5.8 \%)$. One patient (2\%) developed a postoperative CSF leak that resolved with lumbar drain placement. Delayed, symptomatic hyponatremia developed in 3/52 patients (5.8\%). One patient developed new adrenal insufficiency requiring maintenance cortisol replacement therapy, another was found to have SIADH and was treated with fluid restriction, and the third had a transient drop in serum sodium that readily corrected with the administration of normal saline.

\section{Pathological Results}

The most common IPA subtype was nonfunctioning PA (94\%). Of the nonfunctioning IPAs, $71.4 \%$ were positive for alpha subunit, $36.7 \%$ for FSH, $32.7 \%$ for $\mathrm{LH}$, $10.2 \%$ for ACTH, $6.1 \%$ for $\mathrm{GH}, 6.1 \%$ for prolactin, and $4.1 \%$ for TSH; $18.4 \%$ were entirely negative (null cell). In addition, the 3 functional tumors had positive staining for respective hormone subtypes: 2 for prolactin and 1 for GH. The full histopathological results are listed in Table 5.

\section{Clinical Outcomes}

The median follow-up was 67 months, and the mean was 61 months (range 3-204 months). Median imaging follow-up was 55 months with a mean of 61 months (range 0-233 months). One patient with acromegaly was followed up with biochemical measures.

Of the 11 patients who reported headache preoperatively, 8 had recorded postoperative data. Fifty percent of those with preoperative headaches reported improved headaches postoperatively. One of 41 patients (2.4\%) without headaches preoperatively developed a headache postoperatively.

Of the 16 patients who presented with vision loss, $6 / 11$ (54.5\%) patients reported improved vision, 5/11 (45.5\%) reported stable vision, and data were unavailable for the
TABLE 4. Operative data

\begin{tabular}{|c|c|}
\hline Parameter & Value \\
\hline \multicolumn{2}{|l|}{ Surgical procedure } \\
\hline Microscopic transsphenoidal & $50(96.2 \%)$ \\
\hline Endoscopic transsphenoidal & $2(3.8 \%)$ \\
\hline Intraop CSF leak requiring repair & $29(55.8 \%)$ \\
\hline Fat only & $14(26.9 \%)$ \\
\hline Fat \& fascia & $14(26.9 \%)$ \\
\hline Fascia only & $1(1.9 \%)$ \\
\hline Duragen & $1(1.9 \%)$ \\
\hline Lumbar drain & 0 \\
\hline Pedicled nasal septal flap & 0 \\
\hline \multicolumn{2}{|l|}{ Postop imaging } \\
\hline GTR & $27(51.9 \%)$ \\
\hline STR & $24(46.1 \%)$ \\
\hline Not performed & $1(1.9 \%)$ \\
\hline \multicolumn{2}{|l|}{ Postop complications } \\
\hline None & $42(80.8 \%)$ \\
\hline Transient DI & $3(5.8 \%)$ \\
\hline CSF leak & $1(1.9 \%)$ \\
\hline Delayed hyponatremia & $3(5.8 \%)$ \\
\hline Major complication & 0 \\
\hline Pan/hypopituitarism & $2(3.8 \%)$ \\
\hline Other & $1(1.9 \%)$ \\
\hline \multicolumn{2}{|l|}{ Hospital LOS days } \\
\hline Median & 2 \\
\hline Range & $2-7$ \\
\hline
\end{tabular}

LOS = length of stay.

remaining 5 patients. No patients reported worsening of visual symptoms.

Four patients (4/16) had improved endocrine function, including one with remission of acromegaly and two with decreases in prolactin levels to the 30s. One patient with a stalk effect and amenorrhea resumed menstrual periods postoperatively. Four of 38 patients $(10.5 \%)$ with available postoperative endocrine results developed new single-axis hypopituitarism; there were two new cases of hypogonadism, one new hypocortisolism and one new hypothyroidism. One patient $(2.6 \%)$ developed new panhypopituitarism requiring replacement of all pituitary hormones. All patients with new postoperative endocrine dysfunction had invasive tumors. Of the patients with new single-axis dysfunction, 1 had a Knosp grade 4 tumor, 2 had Knosp grade 3 tumors, and 1 had a Knosp grade 2 tumor. Three of the 4 patients also received postoperative radiation, with 2 receiving stereotactic radiosurgery and 1 receiving radiation therapy. The single case of new panhypopituitarism had a Knosp grade 3 tumor and did not have postoperative radiation.

Intraoperative gross-total tumor resection, assessed by surgeon visualization, was achieved in 35 patients $(67 \%)$; however, 8 tumors (23\%) graded as gross-total resection (GTR) at the time of surgery were found on imaging to be 
TABLE 5. Tumor characteristics

\begin{tabular}{lc}
\hline \multicolumn{1}{c}{ Parameter } & Value \\
\hline Size & \\
\hline Macroadenoma & $48(92.3 \%)$ \\
\hline Microadenoma & $1(1.9 \%)$ \\
\hline Not documented & $3(5.8 \%)$ \\
\hline Mean max diameter in mm ${ }^{*}$ & 20.9 \\
\hline Invasiveness & \\
\hline Suprasellar & $42(80.8 \%)$ \\
\hline Sphenoid sinus & $11(21.2 \%)$ \\
\hline Clivus & $1(1.9 \%)$ \\
\hline Cavernous sinus & $29(55.8 \%)$ \\
\hline No invasion & $3(6 \%)$ \\
\hline Hormonal function & \\
\hline Nonfunctioning IPA & $49(94.2 \%)$ \\
\hline Prolactinoma & $2(3.8 \%)$ \\
\hline GH & $1(1.9 \%)$ \\
\hline Immunohistochemistry (nonfunctioning IPAs) & \\
\hline Alpha subunit & $35(71.4 \%)$ \\
\hline FSH & $18(36.7 \%)$ \\
\hline LH & $16(32.7 \%)$ \\
\hline Null cell & $9(18.4 \%)$ \\
\hline ACTH & $5(10.2 \%)$ \\
\hline GH & $3(6.1 \%)$ \\
\hline Prolactin & $3(6.1 \%)$ \\
\hline TSH & $2(4.1 \%)$ \\
\hline
\end{tabular}

${ }^{*}$ Measurement data available in 35 patients.

subtotal resections (STRs). As assessed on postoperative MRI, GTR was achieved in 27/51 patients (53\%) and STR in $24 / 51$ patients $(47 \%)$. There was no significant difference in the extent of resection between asymptomatic and symptomatic patients $(\mathrm{p}=0.57)$. One case, classified as an operative GTR, was subsequently followed biochemically instead of with imaging; therefore, imaging confirmation could not be obtained.

At the latest follow-up, of the 24 patients in whom STR was achieved, 2 (8.3\%) experienced progression after a mean of 45 months (range 23-66 months). One case was treated with medication and radiosurgery, and the other was treated with surgery and radiation therapy. Three (11.1\%) of the 27 cases originally assessed as GTR both surgically and on follow-up imaging recurred after a mean of 104 months (range 80-172 months). Clinical outcomes can be found in Table 6 .

\section{Discussion}

\section{Analysis of Cases}

We report a series of 52 patients surgically treated for IPAs over nearly 2 decades. Despite the availability of practice guidelines, clinical decision-making in patients found to have IPAs poses ongoing challenges. Important factors to consider include patient age, the presence of any
TABLE 6. Clinical outcomes

\begin{tabular}{|c|c|}
\hline Parameter & Value \\
\hline \multicolumn{2}{|l|}{ FU period in mos } \\
\hline Median & 67 \\
\hline Mean & 61 \\
\hline Range & $3-204$ \\
\hline \multicolumn{2}{|l|}{ Headache } \\
\hline Preop headache improved after op & $4 / 8(50.0 \%)$ \\
\hline Preop headache stable after op & $4 / 8(50.0 \%)$ \\
\hline Postop data unavailable & $3 / 11(27.3 \%)$ \\
\hline New-onset headache after op & $1 / 41(2.4 \%)$ \\
\hline \multicolumn{2}{|l|}{ Vision } \\
\hline Improved vision & $6 / 11(54.5 \%)$ \\
\hline Stable vision & $5 / 11(45.6 \%)$ \\
\hline Worsened vision & 0 \\
\hline \multicolumn{2}{|l|}{ Endocrine } \\
\hline Improved endocrine function & $4 / 16(25 \%)$ \\
\hline New hypopituitarism (any single axis) & $4 / 38(10.5 \%)^{*}$ \\
\hline New panhypopituitarism & $1 / 38(2.6 \%)^{*}$ \\
\hline \multicolumn{2}{|l|}{ Additional postop treatments } \\
\hline Radiosurgery & $18(34.6)$ \\
\hline Additional op & $2(3.8 \%)$ \\
\hline Medications & $1(1.9 \%)$ \\
\hline Radiotherapy & $1(1.9 \%)$ \\
\hline Progression after STR & $2 / 24(8.3 \%)$ \\
\hline Mean time to progression in mos & 45 \\
\hline \multicolumn{2}{|l|}{ Treatment of progression } \\
\hline Op + radiation & 1 \\
\hline Radiosurgery + medication & 1 \\
\hline Recurrence after GTR & $3 / 27(11.1 \%)$ \\
\hline Mean time to recurrence in mos & 104 \\
\hline \multicolumn{2}{|l|}{ Treatment of recurrence } \\
\hline Op & 1 \\
\hline Radiosurgery & 2 \\
\hline
\end{tabular}

* All patients with new postoperative single-axis endocrine dysfunction had invasive tumors: Knosp grade 4 (1), grade 3 (2), and grade 2 (1). Three of the 4 required postoperative radiation. The patient with new postoperative panhypopituitarism had a Knosp grade 3 tumor and did not require radiation.

symptoms (even if not the indication for obtaining imaging studies), tumor size or growth, and the results of both visual and endocrine testing. At our tertiary care pituitary center, patients with IPAs comprised $3.1 \%$ of those who underwent transsphenoidal surgery for sellar lesions over almost 2 decades. Our threshold in recommending surgery for this patient population was relatively high, as illustrated by the large mean maximal adenoma diameter (20.9 $\mathrm{mm}$ ) and highly selective clinical rationale for operating on prolactinomas and the lone microadenoma. Though the tumors were found incidentally, detailed workup revealed at least one symptom in a majority of patients $(63.5 \%$ [33/52]).

The safety associated with this surgical intervention is 
supported by the lack of significant major adverse events, including death, permanent neurological morbidity, meningitis, or hemorrhage. Additionally, apart from the single new case of panhypopituitarism, an unfortunate but unavoidable risk of sellar surgery of all types, no long-term morbidity was noted at the last follow-up, with the vast majority of patients having positive clinical outcomes. Similarly, recurrence was infrequent and took place after lengthy follow-up periods, showing that excellent rates of long-term remission or stable disease control are achievable. The results from this retrospective series with its long clinical follow-up averaging more than 4 years support the safety and efficacy of the surgical management of IPA when performed by experienced pituitary surgeons at high-volume centers. Our findings may also aid practitioners when counseling patients regarding the risks and benefits of surgical intervention for IPAs meeting criteria for such treatment.

\section{Role of Neuroimaging in IPAs Over Time}

Over the 2 decades in which this retrospective patient population was treated, the use of routine neuroimaging has increased significantly. ${ }^{1}$ Although the prevalence of IPA remains relatively high, it can often be left untreated if it is asymptomatic and nonfunctional and can instead be observed serially for growth or the development of visual or endocrine deficits. ${ }^{2}$ Despite changes in clinical practice and a greater movement toward observation and nonoperative management of asymptomatic nonfunctional PAs, surgery is a safe and effective option in IPA management, playing a role that will only increase in importance with the greater use of neuroimaging.

\section{Review of Current Guidelines Pertaining to IPA Management}

The Endocrine Society recommends that all patients, regardless of their symptoms, undergo thorough biochemical testing for hormonal irregularities upon discovery of a pituitary incidentaloma of any type. ${ }^{5}$ Screening for hypopituitarism, hyperprolactinemia, and acromegaly is generally accepted, but screening for Cushing's disease remains controversial; Galland et al. contended that Cushing's disease screening is unnecessary in an asymptomatic patient because the lack of symptoms makes Cushing's disease extremely unlikely? In our practice, care is taken to screen for hypocortisolemia in surgical patients and to correct it prior to surgery to prevent a perioperative adrenal crisis.

In our series, 3 patients (5.8\%) had evidence of functional IPAs. This is consistent with a significant amount of the literature published on the overall prevalence of PAs in the general population as well as with past reports of IPA..$^{3,6,7,16}$ Hormonally active PAs are managed according to the generally accepted standards for the treatment of functional PAs, including dopamine agonists for prolactinomas and surgery for other types. ${ }^{67,16,18}$

In addition, all patients with tumors adjacent to or abutting the optic chiasm should undergo a formal visual field examination. In our practice, patients with macroadenomas causing chiasm compression undergo such an examination, regardless of the subjective visual field deficits on physical examination. This practice is informed by anec- dotal evidence that vision loss that does not manifest clinically can manifest on formal examination. Our data here support this as $12 / 22$ patients with formal visual field results available for review had visual deficits even though only 12/52 (23\%) patients were aware of any vision loss at the time of evaluation.

Indications for surgical intervention in patients with pituitary incidentalomas of any type, including IPAs, may include age, presence/absence of medical comorbidities, tumor size, hormonal dysfunction, and vision loss. Evidence of interval tumor growth during conservative monitoring may also be an indication for resection of nonfunctional microadenomas. Formulating an appropriate recommendation and counseling for a patient with a pituitary incidentaloma involves consideration of all of these factors, a discussion pertaining to the risk/benefit ratio of intervention, and consideration of patient preferences.

For patients with nonfunctional microincidentalomas (any mass $<10 \mathrm{~mm}$ ), we recommend a single follow-up scan 1 year after initial presentation and no further followup if the lesion is stable in size at that time; repeat imaging should only occur if symptoms arise. Monitoring of asymptomatic nonfunctional macroincidentalomas (any mass $>10 \mathrm{~mm}$ ) can be performed 6 or 12 months after initial presentation and annually thereafter, though there is variability in the follow-up time-between 3 years and indefinitely., ${ }^{7,17}$ Patients who are not ideal surgical candidates, such as older patients, those with comorbidities, or those with macroincidentalomas abutting the optic apparatus and who may have minor visual field deficits, can be serially monitored with MRI and visual field testing every 6 months.

\section{Review of the Literature}

The characteristics of IPAs vary by study, highlighting the heterogeneous nature of these tumors. Sanno et al. published a large, multicenter, retrospective surgical series of 258 patients with pituitary incidentalomas..$^{18}$ In this group, there were 209 IPAs and the remaining lesions were Rathke's cleft and arachnoid cysts, and the most common reasons for initial neuroimaging were headache (39.5\%), vertigo/tinnitus (15.9\%), head injury (8.9\%), and other brain disease $(7.8 \%)$. Unfortunately, this study did not compile an initial symptom review of all surgical patients for comparison. The average tumor diameter was $26.5 \mathrm{~mm}$, and suprasellar extension was present in 124 cases $(48.1 \%)$. Two hundred fifty-three cases were treated via a transsphenoidal approach, and the authors did not note any significant complications.

Molitch published a review of 10 case series involving 513 IPAs, including 353 macroadenomas and 160 microadenomas. ${ }^{15}$ In this review, the author noted that although only 17 of the microadenomas (10.6\%) had shown growth on repeat imaging by 8 years' follow-up, 85 macroadenomas $(24.1 \%)$ showed growth in the same period. The author also noted that the rate at which macroadenomas are found is higher than expected according to autopsy data and posited that the mass effect alone can result in some of the symptoms that drive the scans to be obtained. Bancos et al. published a review article, in which they came to similar conclusions, citing a nearly 4 -fold (3.3/100 pa- 
tient-years vs 12.5/100 patient-years) increased incidence of growth for macroadenomas versus microadenomas. ${ }^{2}$ Furthermore, no associations between non-modifiable risk factors, such as age, sex, or invasiveness and tumor growth rate, have been conclusively demonstrated. ${ }^{2,15,16}$ Although both studies are limited in their power and conclusions, in general it remains that the growth rate of incidental macroadenoma is substantial from the standpoint of long-term clinical observation, especially for patients presenting with intact or only mildly impaired vision and preserved endocrine function.

Two studies of incidentalomas have proposed that patients with asymptomatic incidentalomas benefit from early surgery in terms of visual outcomes, endocrine outcomes, and extent of resection..$^{12,13}$ We did not observe an improved outcome with regard to the extent of resection in our patient population; however, it is unclear whether our conflicting findings are due to a small sample size or differences in the patient populations selected for surgery. The relative infrequency with which incidentalomas meet criteria for surgical intervention, even at large-volume surgical centers, limits finer analysis of factors contributing to outcomes. Since the referral rate for incidentalomas may be expected to rise given the increasing use of cranial imaging, further study is warranted to better define the population with the best risk/benefit ratio. As our results indicated, however, excellent outcomes can be obtained in a properly screened population.

\section{Study Limitations}

As the current study is primarily a retrospective surgical series, it cannot speak to the overall population of patients counseled in our clinic who were managed conservatively and is therefore limited by the fact that it represents a select group that was eligible for and elected to undergo surgery. Additionally, as our surgical center is a tertiary care referral center without an affiliated emergency room, our study cannot speak to the rate at which incidentalomas are identified, which has been addressed by excellent radiographic and anatomical studies. ${ }^{3,4,10,20}$ This element of selection bias limits our data to patients meeting criteria for surgical intervention and not to all patients with incidentally discovered PAs.

The data set is also somewhat limited by the duration of the study period, over which the surgical technique has changed, notably from microscopic to endoscopic surgery. Despite this transition, our results remain valuable as both approaches have been shown to be efficacious when performed by experienced pituitary surgeons at high-volume centers. ${ }^{8,11}$

Our analysis is further limited by the completeness of patient medical records. The transition to electronic medical records made important elements, such as imaging results and follow-up time, unavailable for certain patients because of an inability to transfer all patient data to the electronic record prior to paper chart disposal.

Finally, our data were sourced from the records of our referral center, and so relied heavily on initial patient evaluations performed by outside referring physicians and laboratories. Because of an inability to control for the variability present between different labs and assays, biochemical data were, for the most part, not included.

\section{Conclusions}

Incidental PAs are common imaging findings during diagnostic evaluations for unrelated reasons or nonspecific symptoms. After initial endocrine and neurosurgical workup, most of these tumors do not require treatment and periodic follow-up is sufficient per established guidelines. However, some IPAs, especially macroadenomas, as well as those demonstrating serial growth and/or causing endocrinopathies, should be evaluated and treated, depending on patient age, comorbidities, and patient preferences. As our series indicates, surgical treatment of IPAs, when appropriate, can be performed safely and effectively at highvolume pituitary centers.

\section{References}

1. Agustsson TT, Baldvinsdottir T, Jonasson JG, Olafsdottir E, Steinthorsdottir V, Sigurdsson G, et al: The epidemiology of pituitary adenomas in Iceland, 1955-2012: a nationwide population-based study. Eur J Endocrinol 173:655-664, 2015

2. Bancos I, Natt N, Murad MH, Montori VM: Evidence-based endocrinology: illustrating its principles in the management of patients with pituitary incidentalomas. Best Pract Res Clin Endocrinol Metab 26:9-19, 2012

3. Ezzat S, Asa SL, Couldwell WT, Barr CE, Dodge WE, Vance ML, et al: The prevalence of pituitary adenomas: a systematic review. Cancer 101:613-619, 2004

4. Famini P, Maya MM, Melmed S: Pituitary magnetic resonance imaging for sellar and parasellar masses: ten-year experience in 2598 patients. J Clin Endocrinol Metab 96:1633-1641, 2011

5. Freda PU, Beckers AM, Katznelson L, Molitch ME, Montori VM, Post KD, et al: Pituitary incidentaloma: an endocrine society clinical practice guideline. J Clin Endocrinol Metab 96:894-904, 2011

6. Freda PU, Wardlaw SL, Post KD: Unusual causes of sellar/ parasellar masses in a large transsphenoidal surgical series. J Clin Endocrinol Metab 81:3455-3459, 1996

7. Galland F, Vantyghem MC, Cazabat L, Boulin A, Cotton F, Bonneville JF, et al: Management of nonfunctioning pituitary incidentaloma. Ann Endocrinol (Paris) 76:191-200, 2015

8. Gao Z, Cai L, Lu J, Wang C, Li Q, Chen J, et al: Expression of stem cell markers and dopamine D2 receptors in human and rat prolactinomas. Med Sci Monit 23:1827-1833, 2017

9. Harris PA, Taylor R, Thielke R, Payne J, Gonzalez N, Conde JG: Research electronic data capture (REDCap) - a metadata-driven methodology and workflow process for providing translational research informatics support. J Biomed Inform 42:377-381, 2009

10. Kleinschmidt-DeMasters BK, Lillehei KO, Stears JC: The pathologic, surgical, and MR spectrum of Rathke cleft cysts. Surg Neurol 44:19-27, 1995

11. Lobo B, Heng A, Barkhoudarian G, Griffiths CF, Kelly DF: The expanding role of the endonasal endoscopic approach in pituitary and skull base surgery: a 2014 perspective. Surg Neurol Int 6:82, 2015

12. Losa M, Donofrio CA, Barzaghi R, Mortini P: Presentation and surgical results of incidentally discovered nonfunctioning pituitary adenomas: evidence for a better outcome independently of other patients' characteristics. Eur J Endocrinol 169:735-742, 2013

13. Messerer M, Dubourg J, Raverot G, Bervini D, Berhouma M, George I, et al: Non-functioning pituitary macro-incidentalomas benefit from early surgery before becoming symptomatic. Clin Neurol Neurosurg 115:2514-2520, 2013

14. Molitch ME: Pituitary incidentalomas. Endocrinol Metab Clin North Am 26:725-740, 1997 
15. Molitch ME: Pituitary tumours: pituitary incidentalomas. Best Pract Res Clin Endocrinol Metab 23:667-675, 2009

16. Orija IB, Weil RJ, Hamrahian AH: Pituitary incidentaloma. Best Pract Res Clin Endocrinol Metab 26:47-68, 2012

17. Randall BR, Kraus KL, Simard MF, Couldwell WT: Cost of evaluation of patients with pituitary incidentaloma. Pituitary 13:383-384, 2010

18. Sanno N, Oyama K, Tahara S, Teramoto A, Kato Y: A survey of pituitary incidentaloma in Japan. Eur J Endocrinol 149:123-127, 2003

19. Serhal D, Weil RJ, Hamrahian AH: Evaluation and management of pituitary incidentalomas. Cleve Clin J Med 75:793801,2008

20. Teramoto A, Hirakawa K, Sanno N, Osamura Y: Incidental pituitary lesions in 1,000 unselected autopsy specimens. Radiology 193:161-164, 1994

\section{Disclosures}

The authors report no conflict of interest concerning the materials or methods used in this study or the findings specified in this paper.

\section{Author Contributions}

Conception and design: Seltzer, Carmichael, Weiss, Zada. Acquisition of data: Seltzer, Wedemeyer. Analysis and interpretation of data: Seltzer, Wedemeyer, Bonney, Carmichael, Zada. Drafting the article: Seltzer, Bonney. Critically revising the article: Seltzer, Wedemeyer, Bonney, Carmichael, Zada. Reviewed submitted version of manuscript: all authors. Approved the final version of the manuscript on behalf of all authors: Seltzer. Statistical analysis: Seltzer, Wedemeyer. Study supervision: Carmichael, Weiss, Zada.

\section{Supplemental Information}

Previous Presentations

A very early iteration of this project was presented at the 6th Annual North American Skull Base Society Meeting held in Scottsdale, Arizona, on February 12-14, 2016. The data set and analysis have been significantly changed and refined since this presentation.

\section{Correspondence}

Justin Seltzer: Keck School of Medicine of USC, Los Angeles, CA.jseltzer@gmail.com 